\title{
NONADDITIVE GAUSSIAN WATERMARKING AND ITS APPLICATION TO WAVELET-BASED IMAGE WATERMARKING
}

\author{
Pierre Moulin and Aleksandar Ivanović
}

\author{
University of Illinois \\ Beckman Inst., Coord. Sci. Lab \& ECE Dept. \\ 405 N. Mathews Ave., Urbana, IL 61801 \\ E-mail: [moulin,ivanovic]@ifp.uiuc.edu
}

\begin{abstract}
This paper extends our recent game-theoretic approach [1] to design and embed watermarks in Gaussian signals in the presence of an adversary. The detector solves a binary hypothesis testing problem. The system is designed to minimize probability of error under the worst-case attack in a prescribed class of attacks. In this paper, the embedder is allowed to filter the host signal and add a watermark, thereby making the scheme nonadditive. The theory is applied to wavelet-based image watermarking. We find that, in this framework, additive watermarks are clearly suboptimal.
\end{abstract}

\section{INTRODUCTION}

Applications of watermarking include copyright protection, document authentication, covert communications, and data embedding. In these applications, a watermark is embedded within a host data set such as text, audio, image, or video. This embedding should be nearly imperceptible and robust against possible manipulations of the watermarked data, in the sense that it should be possible to reliably extract the watermark in a degraded version of the signal. Degradations include operations such as addition of noise, filtering, compression, format conversion, and desynchronization. These degradations could be intentional (due to an adversary) or nonintentional (e.g., due to a lossy communication channel).

We extend recent work [1] on the detection-theoretic aspects of the Gaussian watermarking problem in two ways: first, we enlarge the set of strategies for the watermarker to include signal scaling and filtering, and demonstrate substantial advantages of such strategies; second, we apply the optimal embedding and attack strategies (which are the solution to a maxmin optimization problem) to wavelet-based image watermarking.

WORK SUPPORTED BY NSF GRANTS CCR 00-81268 AND CDA 96-24396.
Notation. We use capital letters to denote random variables and small letters to denote their individual values. The cardinality of a set $\Omega$ is denoted by $|\Omega|$. The Euclidean norm of a vector $x$ is denoted by $\|x\|=\left(\sum_{n} x^{2}(n)\right)^{1 / 2}$. The multivariate Gaussian distribution with mean vector $\mu$ and covariance matrix $R$ is denoted as $\mathcal{N}(\mu, R)$. The mathematical expectation of a random variable $X$ is denoted $\mathbb{E} X$.

\section{MATHEMATICAL MODEL}

Let $s(n), n \in \Omega=\left\{0,1, \cdots, N_{1}-1\right\} \times\left\{0,1, \cdots, N_{2}-1\right\}$ denote the original $N_{1} \times N_{2}$ digital image to be marked, taking values in $\mathbb{R}$ and modeled as a Gaussian random vector $\mathcal{N}\left(0, R_{s}\right)$ whose components are indexed by $n \in \Omega$. A watermark is inserted in $s$, resulting in a marked image $x$, that is made publicly available. The watermark is available at the detector, but $s$ itself is not (blind watermarking). We assume that an adversary (attacker) takes $x$ and produces a degraded image $y$. The decoder has access to $y$ and $k$ but not to $s$ (blind watermarking), and must determine whether the watermark was embedded in $x$ or not.

Assume that the watermarker implements the linear operation:

$$
x=\Phi(s+p),
$$

where $\Phi$ is an $|\Omega| \times|\Omega|$ matrix and $\{p(n), n \in \Omega\} \sim \mathcal{N}\left(0, R_{p}\right)$ is a Gaussian random vector (thought of as a pattern) independent of $s$. We call $w \triangleq x-s=(\Phi-I) s+\Phi p$ the watermark; $w$ depends on $s$ unless $\Phi$ is the identity matrix, $I$. In this case, the watermark is said to be additive. The vector $p$ is available at the detector and is independent of $s$ by construction. The embedding is subject to a constraint on the average squared-error distortion:

$$
\begin{aligned}
D_{w} & \geq|\Omega|^{-1} \mathbb{E}\|X-S\|^{2} \\
& =|\Omega|^{-1} \operatorname{Tr}\left[(\Phi-I) R_{s}(\Phi-I)^{T}+\Phi R_{p} \Phi^{T}\right] .
\end{aligned}
$$

Assume the attacker implements the attack

$$
y=\Gamma x+e,
$$


where $\Gamma$ is an $|\Omega| \times|\Omega|$ matrix and $\{e(n), n \in \Omega\} \sim \mathcal{N}\left(0, R_{e}\right)$, is a Gaussian random vector independent of $x$. The attack is nonadditive, unless $\Gamma=I$. The attack is also subject to a constraint on the average squared-error distortion:

$$
\begin{aligned}
D_{a} \geq|\Omega|^{-1} \mathbb{E}\|Y-S\|^{2} & \\
= & |\Omega|^{-1} \operatorname{Tr}\left[(\Gamma \Phi-I) R_{s}(\Gamma \Phi-I)^{T}\right. \\
& \left.\quad+\Gamma \Phi R_{p} \Phi^{T} \Gamma^{T}+R_{e}\right] .
\end{aligned}
$$

\section{GAME-THEORETIC FORMULATION OF THE WATERMARKING PROBLEM}

It is assumed that the watermark detector knows or is able to learn the statistics of the attack ( $\Gamma$ and $R_{e}$ ); the detector then implements the optimal likelihood ratio test (LRT) [2]. The detector decides between the two hypotheses:

$$
\left\{\begin{array}{l}
H_{0}: Y \sim \mathcal{N}\left(0, R_{y}\right) \\
H_{1}: Y \sim \mathcal{N}\left(\Gamma \Phi p, R_{y}\right)
\end{array}\right.
$$

where $R_{y}=\operatorname{Cov}[Y]=\Gamma \Phi R_{s} \Phi \Gamma^{T}+R_{e}$ is the covariance matrix for the data $Y$ conditioned on $P$ which is available at the detector.

\subsection{Probability of Error}

The probability of error of the LRT is denoted by $P_{e}\left(\Phi, R_{p}, \Gamma, R_{e}\right)$ to indicate its dependency on the statistics of the embedding process and the attack. The watermarker wants to minimize $P_{e}$ and the attacker wants to maximize it. The problem is thus naturally formulated as a game between the information hider and the attacker. The detection game is a minmax problem:

$$
\min _{\Phi, R_{p}} \max _{\Gamma, R_{e}} P_{e}\left(\Phi, R_{p}, \Gamma, R_{e}\right)
$$

where the optimization is subject to the distortion constraints (2) and (4).

Assume equally likely hypotheses. For the test (5), $P_{e}=$ $Q(d / 2)$, where $Q(t) \triangleq(2 \pi)^{-1 / 2} \int_{t}^{\infty} e^{-u^{2} / 2} d u$ is the tail probability of the normal distribution $\mathcal{N}(0,1)$, and [2]

$$
d^{2}=p^{T} \Phi^{T} \Gamma^{T} R_{y}^{-1} \Gamma \Phi p
$$

is twice the Kullback-Leibler divergence between the two Gaussian distributions in (5). The average probability of error is given by

$$
\bar{P}_{e} \triangleq \mathbb{E} P_{e} \approx Q(\bar{d} / 2)
$$

where $\bar{d}^{2} \triangleq \mathbb{E} d^{2}=\operatorname{Tr}\left[R_{p} \Phi^{T} \Gamma^{T} R_{y}^{-1} \Gamma \Phi\right]$ is the average value of $d^{2}$. The approximation is accurate if the distribution of $d^{2}$ is narrowly concentrated around its mean $\bar{d}^{2}$. Due to the law of large numbers, this property holds under mild technical assumptions. Hence we use the averaged distance $\bar{d}^{2}$ as our design criterion, and seek

$$
\max _{\Phi, R_{p}} \min _{\Gamma, R_{e}} \bar{d}^{2}\left(\Phi, R_{p}, \Gamma, R_{e}\right),
$$

which is equivalent to (6).

\subsection{Solution of Maxmin Problem (6)}

The solution to the minmax problem has been derived analytically [3] following a procedure similar to [1]. The derivation is not included here due to space constraints. The solution possesses some remarkable properties.

1. The optimal strategies for both the watermarker and the attacker consist in applying diagonal processors to the KLT coefficients of the signal, as shown in Fig. 1. The KLT diagonalizes the covariance matrix $R_{s}$ of the host signal. The optimization problem (6) is reduced to

$$
\max _{F, \lambda_{p}} \min _{G, \lambda_{e}} \bar{d}^{2}\left(F, \lambda_{p}, G, \lambda_{e}\right),
$$

where $F(i)$ and $G(i), \in \Omega$ are viewed as filters, and $\lambda_{p}(i)$ and $\lambda_{e}(i), i \in \Omega$, are variances of the pattern and attacker noise, respectively.

2. The maxmin problem above is further reduced to one involving optimal power allocations $\delta_{w}(i)$ and $\delta_{a}(i), i \in$ $\Omega$ for the watermarker and the attacker, respectively. These are distortion profiles in channel $i$. Given $\delta_{w}(i)$, the optimal $F(i), \lambda_{p}(i)$ are given by the Gaussian test channel with distortion $\delta_{w}(i)$ from rate-distortion theory. Given $\delta_{a}(i)$, the optimal $G(i), \lambda_{e}(i)$ are given by the Gaussian test channel with distortion $\delta_{a}(i)-$ $\delta_{w}(i)$

3. Although the two properties above are similar to those satisfied by the solution to the Gaussian data-hiding problem [4], the optimal power allocations are completely different.

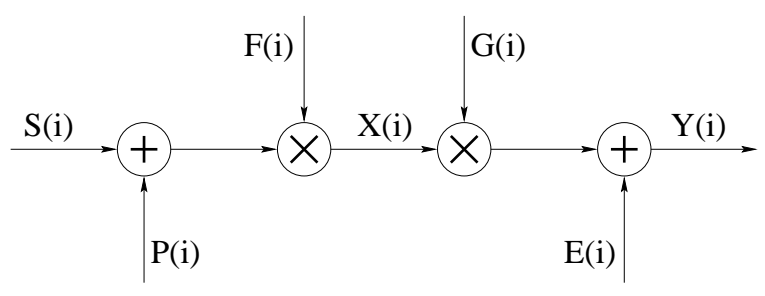

Fig. 1. KLT-domain processing for watermarker and attacker in Gaussian watermarking problem $(i \in \Omega)$.

The representation of the vectors $s, p, x, e, y$ in the KLT basis is $\tilde{s}(i), \tilde{p}(i), \tilde{x}(i), \tilde{e}(i), \tilde{y}(i), i \in \Omega$. 


\section{WAVELET IMAGE WATERMARKING}

In this section, we use a local Gaussian model for image wavelet coefficients to compute estimates of the probability of error and the optimal distributions for the watermarker and attacker. The wavelet transform is viewed as an approximate KLT. In all experiments, we used Daubechies' 9/7 wavelets and 4 decomposition levels (10 subbands). In order to facilitate comparisons, all images are normalized in such a way that the per-pixel energy of their wavelet coefficients (not including coarse-band coefficients - see below) is unity. The normalization factor for the energies is 0.0056 for Barbara, 0.0076 for Baboon, 0.0029 for Goldhill, and 0.0025 for Lena. Let $\tilde{s}(i), \tilde{p}(i), \tilde{x}(i), \tilde{y}(i), i \in \Omega$ respectively denote the wavelet coefficients of the host image, the watermark pattern, the watermarked image, and the output of the attack.

\subsection{Statistical Model for Wavelet Coefficients}

Several statistical models for image wavelet coefficients have been proposed in the literature. To capture the spatial clustering of significant wavelet coefficients, LoPresto et. al. [6] proposed the Estimation-Quantization (EQ) model. We use a simplified version of this model, which defines a local Gaussian model. This model has been successfully used in compression and denoising applications [7], and in evaluations of the data-hiding capacity of image sources [5].

\subsection{EQ Model}

We model the wavelet coefficients as independent Gaussian random variables having zero mean and unequal variances. The variance field in each subband is assumed to be slowly changing. The encoder, attacker and decoder are assumed to know or be able to estimate this variance field.

For each wavelet coefficient $\tilde{S}(i)$, an estimate of $\lambda_{s}(i)$ is formed based on a $5 \times 5$ square window $\mathcal{N}(i)$ centered at location $i$. The estimate of $\lambda_{s}(i)$ is given by $\hat{\lambda}_{s}(i)=$ $\frac{1}{25} \sum_{j \in \mathcal{N}(i)}|\tilde{S}(j)|^{2}$. The performance of the watermarking detector will depend, to some extent, on the quality of the variance field estimator.

The watermark is embedded only in the detail subbands. Information is not embedded in approximation coefficients, for which a local Gaussian model would be less accurate.

\subsection{Distortion Levels}

We need to find realistic values for the distortion levels $D_{w}$ and $D_{a}$. We find it convenient to specify $D_{w}$ and the distortion ratio

$$
\gamma \triangleq \frac{D_{a}}{D_{w}}
$$

Due to the existence of threshold effects in the human visual system, the addition of a visual pattern to an image is not noticeable when the pattern intensity is low enough. The just noticeable $(\mathrm{JN})$ pattern intensity level depends both on the image and on the pattern. We make the standard assumption that the visibility of a random pattern depends primarily on its second-order statistics [8]. When considering a particular application, the watermarker would often assume a target $D_{a}$ for the attacker and select $D_{w}$ so that the watermarks are slightly below a JN level $D_{w}^{*}\left(D_{a}\right)$. We have experimentally determined the curves $D_{a}^{*}\left(D_{w}\right)$ and $D_{w}^{*}\left(D_{a}\right)$ for Lena, see Table 1.

\subsection{Detection Performance}

Optimal watermarks for Lena are shown in Fig. 2 for several values of $D_{w}$ and $D_{a}$. The values of $D_{w}$ are at the JN level for the particular optimal patterns considered here. (Recall that the statistics of these patterns depend on $D_{a}$, therefore the JN levels also depend on $D_{a}$.) It is interesting to look at cases when the attacker is allowed to introduce significantly more distortion than the watermarker at that figure. The watermark in case $\gamma=10$ is spread out over a wider area because the attacker has more jamming power. To capture this localization property we define three quantities:

Set of Watermarked Pixels $\Omega_{w} \triangleq\{n \in \Omega: p(n) \neq 0\}$. Percentage of Active Pixels $P A P=100 \frac{\left|\Omega_{w}\right|}{|\Omega|}$, i.e., percentage of watermarked image pixels.

Percentage of Active Coefficients $P A C=\frac{100}{|\Omega|}\{\# i: \tilde{P}(i) \neq$ $0\}$, i.e., percentage of watermarked wavelet coefficients.

Subjective evaluation of watermarks and attacks can be supplemented with objective distortion measures such as SNRs. However, the standard SNR may be a deceptive measure because it could be high while the distortion in the critical region $\Omega_{w}$ is high. For this reason we also consider an SNR restricted to $\Omega_{w}$ :

$$
S N R_{w}\left(\Omega_{w}\right) \triangleq \frac{\sum_{n \in \Omega_{w}}[s(n)-\mu]^{2}}{\sum_{n \in \Omega_{w}} p^{2}(n)}
$$

where $\mu$ is the average of $s(n)$ over the region $\Omega_{w}$. Clearly, $S N R_{w}\left(\Omega_{w}\right) \leq S N R_{w}(\Omega)$. Likewise, after attack, we have an area $\Omega_{a}$ of pixels that have been modified by the watermarking process and/or the attack. Typically $\Omega_{a}$ is larger than $\Omega_{w}$. We define

$$
\operatorname{SNR}_{a}\left(\Omega_{a}\right) \triangleq \frac{\sum_{n \in \Omega_{a}}[s(n)-\mu]^{2}}{\sum_{n \in \Omega_{a}}[y(n)-s(n)]^{2}}
$$

where $\mu$ is the average of $s(n)$ over the region $\Omega_{a}$.

Table 2 shows $P A P, P A C$ and SNR values for watermarking of four test images, using JN watermarks. Table 3 shows $P_{e}$ for the optimal nonadditive scheme as well as for the optimal additive scheme [1]. It is seen that relaxing the 
additivity constraint reduces $P_{e}$ by several orders of magnitude.

Remark. The watermarker finds it generally useful to scale down the host signal components to increase the efficiency of the detector while the attacker has an interest in scaling down the watermarked signal components.

Acknowledgment. The authors express their deep gratitude to M. K. Mihçak for co-designing the maxmin optimization algorithm.

\begin{tabular}{|c||c|c||c|c|}
\hline \multicolumn{1}{|c||}{} & \multicolumn{2}{c||}{ JN watermark } & \multicolumn{2}{c|}{ JN attack } \\
\hline$\gamma$ & $D_{w}^{*}\left(D_{a}\right)$ & $D_{a}$ & $D_{w}$ & $D_{a}^{*}\left(D_{w}\right)$ \\
\hline \hline $3 / 2$ & $1 \times 10^{-5}$ & $1.5 \times 10^{-5}$ & $1 \times 10^{-5}$ & $1.5 \times 10^{-5}$ \\
\hline 2 & $1 \times 10^{-5}$ & $2 \times 10^{-5}$ & $5 \times 10^{-6}$ & $1 \times 10^{-5}$ \\
\hline 3 & $5 \times 10^{-6}$ & $1.5 \times 10^{-5}$ & $2.5 \times 10^{-6}$ & $7.5 \times 10^{-6}$ \\
\hline 4 & $5 \times 10^{-6}$ & $2 \times 10^{-5}$ & $5 \times 10^{-7}$ & $2.5 \times 10^{-6}$ \\
\hline 5 & $5 \times 10^{-6}$ & $2.5 \times 10^{-5}$ & $5 \times 10^{-7}$ & $2.5 \times 10^{-6}$ \\
\hline 10 & $5 \times 10^{-5}$ & $5 \times 10^{-4}$ & $1 \times 10^{-7}$ & $1 \times 10^{-6}$ \\
\hline
\end{tabular}

Table 1. JN distortion levels for watermarks and attacks on Lena as a function of distortion ratio $\gamma$.

\begin{tabular}{|l||c|c|c|c|c|c|c|}
\hline Image & $D_{w}^{*}$ & $P A P$ & $P A C$ & $S N R_{w}$ & $S N R_{w}$ & $S N R_{a}$ & $S N R_{a}$ \\
{$[\%]$} & {$[\%]$} & $(\Omega)[\mathrm{dB}]$ & $\left(\Omega_{w}\right)[\mathrm{dB}]$ & $(\Omega)[\mathrm{dB}]$ & $\left(\Omega_{a}\right)[\mathrm{dB}]$ \\
\hline \hline Lena & $1 \times 10^{-5}$ & 3.74 & 1.06 & 35.9 & 16.3 & 34.0 & 15.0 \\
\hline Baboon & $1 \times 10^{-5}$ & 6.5 & 1.5 & 34.6 & 20.0 & 32.5 & 18.5 \\
\hline Goldhill & $5 \times 10^{-5}$ & 7.38 & 2.8 & 29.8 & 19.8 & 28.1 & 18.5 \\
\hline Barbara & $1 \times 10^{-5}$ & 7.96 & 2.0 & 37.2 & 25.1 & 34.9 & 23.3 \\
\hline
\end{tabular}

Table 2. $S N R s$ and percentages of active pixels (PAP) and coefficients (PAC) using JN watermarks $(\gamma=2)$.

\begin{tabular}{|l||c|c||c|c|}
\hline \multicolumn{1}{|l||}{ Image } & \multicolumn{2}{c||}{$P_{e}\left(D_{a}=2 D_{w}\right)$} & \multicolumn{2}{c|}{$P_{e}\left(D_{a}=10 D_{w}\right)$} \\
\hline WM & $\begin{array}{c}\text { optimal } \\
\text { nonadditive }\end{array}$ & $\begin{array}{c}\text { optimal } \\
\text { additive }\end{array}$ & $\begin{array}{c}\text { optimal } \\
\text { nonadditive }\end{array}$ & $\begin{array}{c}\text { optimal } \\
\text { additive }\end{array}$ \\
\hline \hline Lena & $1.8 \times 10^{-24}$ & $1.0 \times 10^{-20}$ & $3.2 \times 10^{-13}$ & $6.8 \times 10^{-13}$ \\
\hline Boat & $9.5 \times 10^{-35}$ & $5.7 \times 10^{-30}$ & $1.8 \times 10^{-21}$ & $7.7 \times 10^{-21}$ \\
\hline
\end{tabular}

Table 3. $P_{e}$ for two images, $D_{w}=10^{-5}$.

\section{REFERENCES}

[1] P. Moulin and A. Ivanović, "Game-Theoretic Analysis of Watermark Detection," IEEE Int. Conf. on Image Processing, Thessaloniki, Greece, Oct. 2001.

[2] H. V. Poor, An Introduction to Detection and Estimation Theory, Springer-Verlag, 2nd Ed., 1994.
[3] P. Moulin and A. Ivanović, "The Spread-Spectrum Watermark Embedding Game," preprint, Feb. 2002.

[4] P. Moulin and M. K. Mihçak, "The Parallel-Gaussian Watermarking Game," UIUC CSL Tech. Rep. ENG-012214, June 2001; revised, Jan. 2002. Available from www. ifp.uiuc.edu/ moulin/Papers/.

[5] P. Moulin, M. K. Mihçak and G.-I. Lin, "An InformationTheoretic Model for Image Watermarking," IEEE Int. Conf. on Image Processing, Vancouver, BC, Sep. 2000.

[6] S. LoPresto, K. Ramchandran and M. T. Orchard, 'Image Coding based on Mixture Modeling of Wavelet Coefficients and a Fast Estimation-Quantization Framework," Proc. Data Compression Conference 97, Snowbird, Utah, pp. 221-230, 1997.

[7] M. K. Mihçak, I. Kozintsev, K. Ramchandran and P. Moulin, "Low-Complexity Image Denoising Based on Statistical Modeling of Wavelet Coefficients," IEEE Signal Processing Letters, Vol. 6, No. 12, pp. 300-303, Dec. 1999.

[8] B. Julesz et al., "Inability of Humans to Discriminate Between Visual Textures that Agree in Second Order Statistics — Revisited," Perception, Vol. 2, pp. 391-405, 1973.

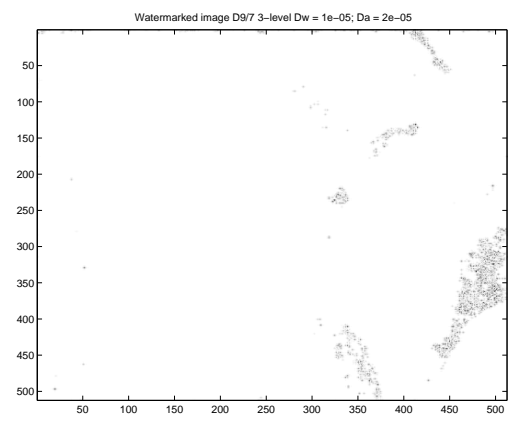

(a) watermark, $D_{w}=10^{-5}, D_{a}=2 D_{w}, P_{e}=1.89 \times 10^{-24}$

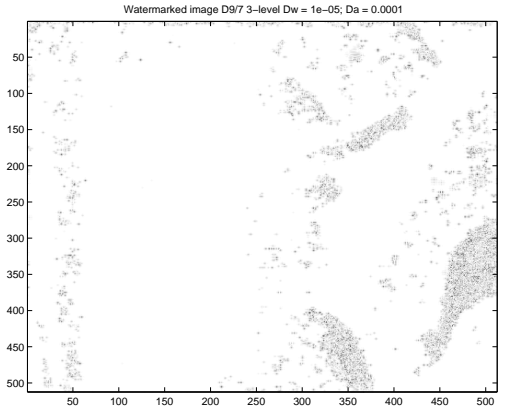

(b) watermark, $D_{w}=10^{-5}, D_{a}=10 D_{w}, P_{e}=3.25 \times 10^{-13}$

Fig. 2. Optimal watermarks for Lena using Daubechies' 9/7 filters and different values of $D_{w}$ and $D_{a}$ 